Review Article

\title{
Effect of Ketamine/Propofol Admixture on Peri-Induction Hemodynamics: A Systematic Review and Meta-Analysis
}

\author{
Nathan J. Smischney ${ }^{1},{ }^{1,2}$ Mohamed O. Seisa, ${ }^{2,3}$ Allison S. Morrow, ${ }^{3}$ Oscar J. Ponce, ${ }^{3,4}$ \\ Zhen Wang, ${ }^{3}$ Muayad Alzuabi, ${ }^{3}$ Katherine J. Heise, ${ }^{1}$ and Mohammad H. Murad ${ }^{3,5}$ \\ ${ }^{1}$ Department of Anesthesiology and Perioperative Medicine, Mayo Clinic, 200 First St. SW, Rochester, MN 55905, USA \\ ${ }^{2}$ HEModynamic and AIRway Management Group, Mayo Clinic, 200 First St. SW, Rochester, MN 55905, USA \\ ${ }^{3}$ Evidence-Based Practice Center, Mayo Clinic Robert D. and Patricia E. Kern Center for the Science of Health Care Delivery, \\ Mayo Clinic, 200 First St. SW, Rochester, MN 55905, USA \\ ${ }^{4}$ Universidad Peruana Cayetano Heredia, Av. Honorio Delgado 430, San Martin de Porres 15102, Lima, Peru \\ ${ }^{5}$ Division of Preventive, Occupational, and Aerospace Medicine, Mayo Clinic, 200 First St. SW, Rochester, MN 55905, USA
}

Correspondence should be addressed to Nathan J. Smischney; smischney.nathan@mayo.edu

Received 17 February 2020; Accepted 15 April 2020; Published 8 May 2020

Academic Editor: Enrico Camporesi

Copyright (c) 2020 Nathan J. Smischney et al. This is an open access article distributed under the Creative Commons Attribution License, which permits unrestricted use, distribution, and reproduction in any medium, provided the original work is properly cited.

To evaluate the effectiveness of an admixture of ketamine and propofol on peri-induction hemodynamics during airway manipulation, we searched electronic databases of randomized controlled trials from January 1, 2000, to October 17, 2018. Trial screening, selection, and data extraction were done independently by two reviewers with outcomes pooled across included trials using the random-effects model. We included 10 randomized trials (722 patients, mean age of 53.99 years, 39.96\% female). American Society of Anesthesiologists physical status was reported in 9 trials with classes I and II representing the majority. Ketamine/propofol admixture was associated with a nonsignificant increase in heart rate (weighted mean difference, 3.36 beats per minute ( $95 \% \mathrm{CI},-0.88,7.60), I^{2}=88.6 \%$ ), a statistically significant increase in systolic blood pressure (weighted mean difference, $\left.9.67 \mathrm{mmHg}(95 \% \mathrm{CI}, 1.48,17.86), I^{2}=87.2 \%\right)$, a nonsignificant increase in diastolic blood pressure (weighted mean difference, $\left.2.18 \mathrm{mmHg}(95 \% \mathrm{CI},-2.82,7.19), I^{2}=73.1 \%\right)$, and a nonsignificant increase in mean arterial pressure (weighted mean difference, $\left.3.28 \mathrm{mmHg}(95 \% \mathrm{CI},-0.94,7.49), I^{2}=69.9 \%\right)$ compared to other agents. The risk of bias was high and the certainty of evidence was low. In conclusion, among patients undergoing airway manipulation and needing sedation, the use of a ketamine/propofol admixture may be associated with better hemodynamics compared to nonketamine/propofol sedation. This trial is registered with CRD42019125725.

\section{Introduction}

Peri-intubation hypotension, defined by either systolic blood pressure or mean arterial pressure (MAP) below a certain threshold (i.e., $<90 \mathrm{mmHg}$ or $<65 \mathrm{mmHg}$ ) or the introduction of vasopressors, has been recognized as a potential target area for research given its association with patientcentered outcomes. For example, peri-intubation hypotension has been associated with both increased length of stay and mortality [1-3]. Furthermore, this association has been identified not only in the critically ill but also in elective surgical patients $[4,5]$. Several studies indicate that the frequency with which peri-intubation hypotension occurs is fairly high, with one report indicating an incidence of greater than $80 \%[1,2,5,6]$. However, the data presented on the frequency is dependent on definitions currently used in the literature for which no standard consensus exists. Perhaps, the best evidence for peri-intubation hypotension incidence in the critically ill comes from Green and colleagues [7]. They performed a systematic review of emergent intubations performed outside the operating room and found that the incidence of peri-intubation hypotension ranged from 5 to 
440 cases per 1,000 intubations $(0.5-44 \%)$ with a pooled estimate of 110 cases per 1,000 intubations (11\%) [7]. Thus, peri-intubation hypotension is not uncommon, and given the associations observed in the literature, prevention of peri-intubation hypotension is likely to improve patientcentered outcomes.

Several putative risk factors have been implicated in the pathway to peri-intubation hypotension. Age, illness severity, and preintubation hemodynamic derangement have consistently been implicated in the development of peri-intubation hypotension $[2,3,8,9]$. One modifiable risk factor potentially leading to peri-intubation hypotension is the choice of intravenous anesthetics with some anesthetics (i.e., propofol and barbiturates) more likely to lead to peri-intubation hypotension than others (i.e., etomidate and ketamine) [10]. Lately, a novel intravenous anesthetic admixture has gained popularity based on potential hemodynamic preservation postadministration [11-13]. The admixture involves the combination of propofol with its vasodilatory effects balanced by the vasoconstricting properties of ketamine [10].

The majority of studies on ketamine/propofol admixture have evaluated critically ill patients in the emergency department with the evidence demonstrating a potential sparing effect on hemodynamics along with improved pain relief and sedation quality. These studies have evaluated ketamine/propofol admixture from the standpoint of a continuous infusion for procedural sedation and analgesia [14-16]. There have been a couple of systematic reviews on ketamine/propofol admixture sedation, demonstrating that ketamine/propofol admixture appears safe and efficacious for procedural sedation and analgesia and is possibly better than propofol only at reducing cardiorespiratory problems $[17,18]$. The wealth of the evidence above has mainly focused on ketamine/propofol admixture use in terms of infusions for procedural sedation and analgesia. There are limited studies addressing the potential hemodynamic preservation effects of the admixture when administered as an induction agent for endotracheal intubation. Given the above associations between peri-intubation hypotension and increased patient morbidity and mortality, and the mounting evidence with ketamine/propofol admixture as an agent that allows potential maintenance of hemodynamics when administered for endotracheal intubation, our aim was to perform a systematic review and meta-analysis on the hemodynamic effects of ketamine/propofol admixture when administered as an induction agent for airway manipulation such as endotracheal intubation.

\section{Methods}

We used the Preferred Reporting Items for Systematic Reviews and Meta-analyses (PRISMA) 2015 statement to report the trial [19-22]. All reviews were conducted by two independent reviewers (MS and AM). Data collection was performed from January 1, 2000, to October 17, 2018. A formal protocol does not exist for this systematic review and meta-analysis. The review was registered with the International Prospective Register of Systematic Reviews (CRD42019125725).
2.1. Eligibility Criteria. Randomized controlled trials published in peer-reviewed journals and written in English were eligible if they included the following: (1) adult patients who received ketamine/propofol admixture as an induction agent in procedural areas and underwent airway manipulation such as endotracheal intubation or supraglottic device placement; (2) a comparison to other induction agents including propofol, ketamine, etomidate, and sodium thiopental or any other combination; and (3) a report of hemodynamic effects (heart rate, systolic and diastolic blood pressure, and MAP) during the first 10 minutes after the induction of general anesthesia in American Society of Anesthesiologists I II, III, and IV patients. The intervention must have equal or similar doses (e.g., ketamine/propofol admixture $1: 1$ or $1: 2$ ratios). No restrictions were placed on trial location, clinical procedure, or patient severity. We excluded pediatric patients, patients who did not undergo induction of anesthesia, or patients who did not undergo airway manipulation. We also excluded observational studies, review articles, erratum, letters, and notes.

2.2. Data Sources and Search Strategies. A comprehensive search of several databases was conducted by a medical reference librarian. The databases included Ovid MEDLINE, Epub Ahead of Print, Ovid Medline In-Process and Other Non-Indexed Citations, Ovid MEDLINE, Ovid EMBASE, Ovid Cochrane Central Register of Controlled Trials, Ovid Cochrane Database of Systematic Reviews, and Scopus. Controlled vocabulary supplemented with keywords was used to search for the trials. We limited our search from January 1, 2000, to October 17, 2018. The search strategy is listed in Table 1.

2.3. Trial Selection. Reviewers, working independently, screened abstracts and titles for eligibility using the above inclusion and exclusion criteria. Full-text articles were then further screened using the same criteria. At the level of fulltext screening, any disagreements were resolved by consensus between the two reviewers (MS and AM) or by consulting a third reviewer (NJS).

2.4. Outcomes. The primary outcomes were hemodynamics (heart rate, systolic and diastolic blood pressure, and MAP) at 5 and 10 minutes following intravenous anesthetic administration. The secondary outcome of interest was pain score as assessed by the visual analog scale (VAS) during the 24 hours postdrug administration.

2.5. Methodological Quality and Certainty of Evidence. The risk of bias was assessed using the Cochrane Collaboration's tool for randomized clinical trials. We assessed random sequence generation, allocation concealment, blinding of providers, outcome assessors, and patients, incomplete outcome data, selective outcome reporting (based on the availability of protocol and inclusion of all prespecified outcomes), and other sources of bias (conflict of interest, source funding, risk of bias due to deviations from 
TABLE 1: Database search strategy.

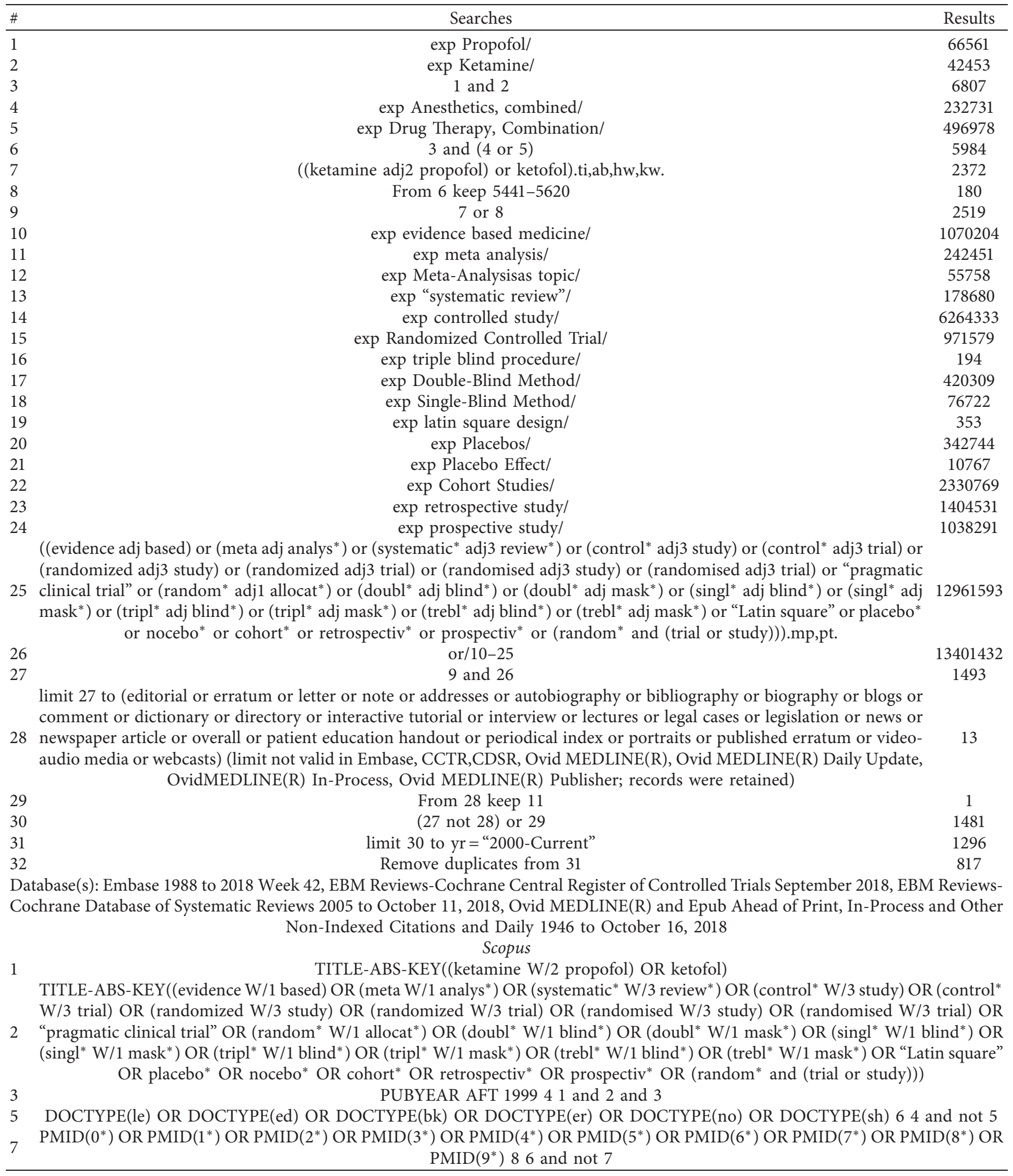

the intended interventions, etc.). Disagreements were resolved by consensus between two reviewers (MS and AM). The overall certainty across trials for each outcome was appraised by discussion between the two reviewers using the grading of recommendations assessment, development, and evaluation (GRADE) approach. Using the grading of recommendations assessment, development, and evaluation approach, randomized trials, as in this systematic review, would provide a starting level of certainty that is high. This level can be downgraded based on the risk of bias of the 
individual trials, inconsistency in the results, indirectness, imprecision, and other considerations to provide a global assessment of the certainty warranted by the body of evidence [23].

2.6. Data Extraction. Two reviewers (MS and AM) independently extracted the following information from each trial: author, publication year, patient characteristics, intervention, comparison, and outcomes.

2.7. Data Synthesis and Statistical Analysis. We extracted or calculated the weighted mean difference (WMD) for continuous outcomes and relative risk for binary outcomes with associated 95\% confidence intervals (CIs) from the included studies. Outcomes without measures of variations (e.g., standard deviation, standard error, and CI) were not included in the meta-analysis. The DerSimonian and Laird random-effects models were used to generate combined effects [24]. A two-sided $P$ value of less than or equal to 0.05 was used to determine significance for the secondary outcome. To evaluate heterogeneity, we calculated the $I^{2}$ statistic $[25,26]$ and heterogeneity $P$ values, where $I^{2}$ more than $50 \%$ or a $P$ value of less than 0.05 suggests high heterogeneity. All statistical analyses were performed using Stata, version 15.0 (StataCorp 2017, College Station, Texas, United States).

\section{Results}

3.1. Trial Inclusion. The searches identified 820 trials. After excluding the irrelevant trials, 33 full-text articles were assessed for eligibility, of which 10 randomized controlled trials $(30 \%)$ met all criteria and were included in data analysis $(n=722)$ (Figure 1$)$. All the trials were reported in full-length journal articles.

3.2. Trial Characteristics. There were a total of 10 trials with 722 patients included. The number of patients in each trial ranged from 40 to 100 (mean, 72.22) with a mean age of 53.99 years. The proportion of participants who were female ranged from 0 to $100 \%$ (mean, 39.96\%). The American Society of Anesthesiologists physiologic status among participants ranged from I to III. Publication dates ranged from 2000 to 2018 (median, 2011 (all trials published after 2000)). The included trials were conducted in several different countries (four trials in Turkey, three trials in Iran, and one trial from India, Japan, Egypt, and United States). The characteristics of the included trials with dosing are summarized in Table 2.

3.3. Risk of Bias. Overall, the risk of bias of included trials was high due to inadequate allocation concealment (7/10, $70 \%)$, partial blinding of outcome assessor (4/10, 40\%), and selective reporting $(9 / 10,90 \%)$. The risk of summary bias is provided in Table 3 .

3.4. Heart Rate. Heart rate at 5 minutes was reported in six trials $(n=465)[11,28-31,34]$, and three trials $(n=244)$
$[11,31,34]$ reported heart rate at 10 minutes. All trials assessed ketamine/propofol admixture, four compared to propofol and two compared to etomidate at 5 minutes and three compared to propofol at 10 minutes. Patients in the ketamine/propofol admixture group had a nonsignificant increase in heart rate at 5 minutes (WMD, 3.36 beats per minute ( $95 \% \mathrm{CI},-0.88$ to 7.60$), I^{2}=88.6 \%$ ) and 10 minutes (WMD, 0.36 beats per minute (95\% CI, -2.57 to 3.29 ), $I^{2}=69.9 \%$ ) compared to patients in the nonketamine/propofol admixture group (Table 4 and Figures 2(a) and 2(b)).

3.5. Systolic Blood Pressure. Systolic blood pressure at 5 minutes was reported in five trials $(n=385)[11,28-31]$ and two trials $(n=164)[11,31]$ reported systolic blood pressure at 10 minutes. All trials assessed ketamine/propofol admixture, three compared to propofol and two compared to etomidate at 5 minutes and two compared to propofol at 10 minutes. Ketamine/propofol admixture was associated with a statistically significant increase in systolic blood pressure at 5 minutes when compared to nonketamine/propofol admixture intravenous anesthetics (WMD, $9.67 \mathrm{mmHg}(95 \%$ CI, 1.48 to 17.86 ), $I^{2}=87.2 \%$ ). At 10 minutes, patients in the ketamine/propofol admixture group had a nonsignificant increase in systolic blood pressure compared to patients in the nonketamine/propofol admixture group (WMD, $4.56 \mathrm{mmHg}$ (95\% CI, -1.09 to 10.20$), I^{2}=0.0 \%$ ) (Table 4 and Figures 3(a) and 3(b)).

3.6. Diastolic Blood Pressure. Diastolic blood pressure at 5 minutes was reported in four trials $(n=305)[11,28-30]$ and one trial $(n=84)$ [11] reported diastolic blood pressure at 10 minutes. All trials assessed ketamine/propofol admixture, two compared to propofol and two compared to etomidate at 5 minutes and one trial compared to propofol at 10 minutes. Patients in the ketamine/propofol admixture group had a nonsignificant increase in diastolic blood pressure at 5 minutes (WMD, $2.18 \mathrm{mmHg}$ (95\% CI, -2.82 to 7.19 ), $I^{2}=73.1 \%$ ) compared to patients in the nonketamine/propofol admixture group. At 10 minutes, there was a statistically significant increase in diastolic blood pressure in the ketamine/propofol admixture group compared to the nonketamine/propofol admixture group (WMD, $4.80 \mathrm{mmHg}$ (95\% CI, 0.24 to 9.36$), I^{2}=$ not applicable) (Table 4 and Figures 4(a) and 4(b)).

3.7. Mean Arterial Pressure. Mean arterial pressure at 5 minutes was reported in four trials $(n=345)[11,28,30,34]$ and 2 trials $(n=164)[11,34]$ reported MAP at 10 minutes. All trials assessed ketamine/propofol admixture, two compared to propofol and two compared to etomidate at 5 minutes and two compared to propofol at 10 minutes. Patients in the ketamine/propofol admixture group had a nonsignificant increase in MAP at 5 minutes (WMD, $3.28 \mathrm{mmHg}(95 \% \mathrm{CI},-0.94$ to 7.49$\left.), I^{2}=69.9 \%\right)$ and 10 minutes (WMD, $4.08 \mathrm{mmHg}(95 \% \mathrm{CI},-0.22$ to 8.39 ), $I^{2}=41.4 \%$ ) compared to patients in the nonketamine/propofol admixture group (Table 4 and Figures 5(a) and 5(b)). 


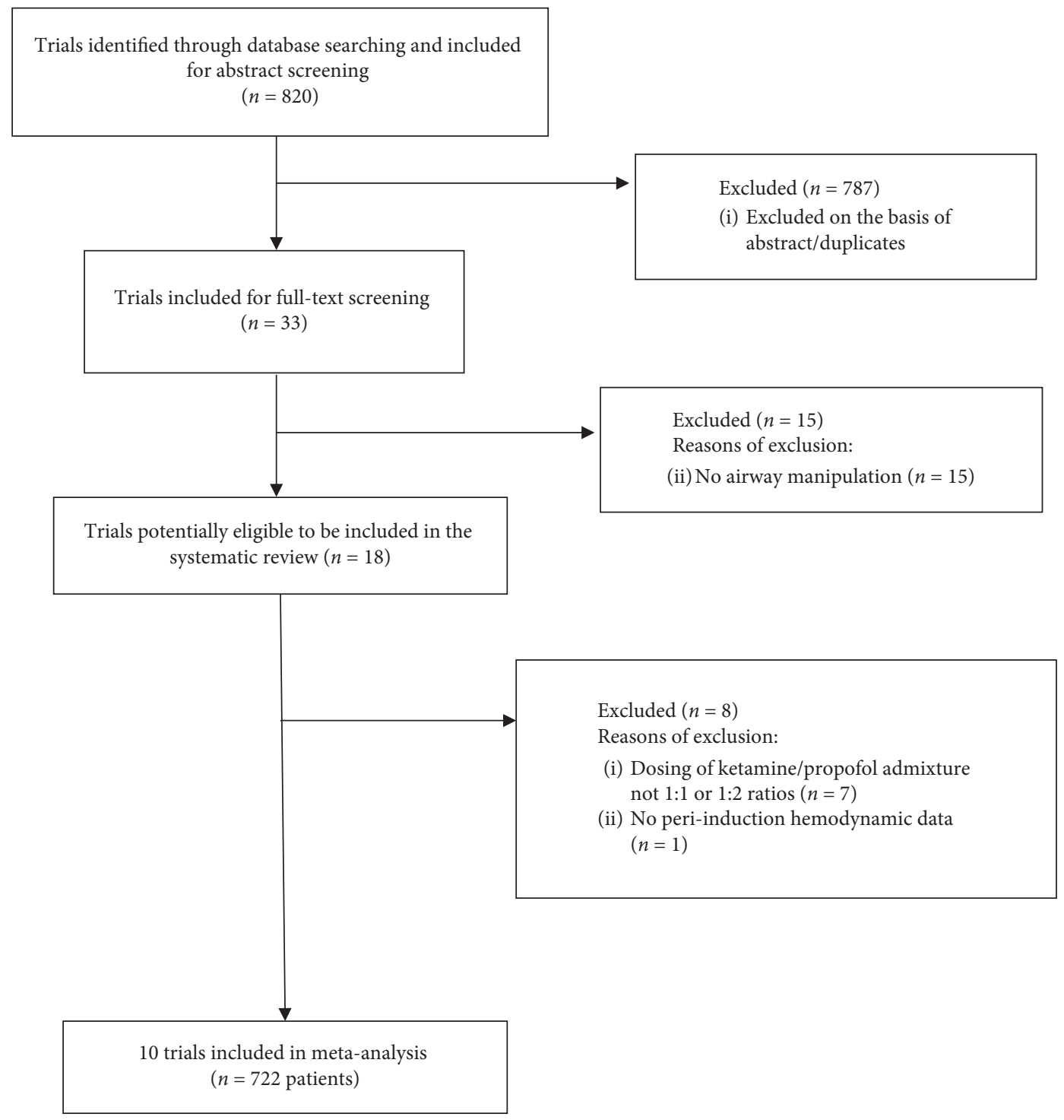

Figure 1: Trial selection.

3.8. Pain. Only one trial assessed postprocedural pain by VAS $(n=60)$ [27]. Twenty-six patients in the propofol plus dexmedetomidine group and all patients in the ketamine/ propofol admixture group (30 patients) had VAS 1-2 (difference between groups, $P=0.12$ ). Four patients in the propofol plus dexmedetomidine group had higher pain scores (VAS 3-5) whereas no patients in the ketamine/ propofol admixture group had high pain scores (VAS 3-5, difference between groups, $P \leq 0.05)$.

\section{Discussion}

The present systematic review and meta-analysis demonstrate that ketamine/propofol admixture results in a potentially better hemodynamic profile as compared to other agents used for induction of anesthesia. In particular, systolic blood pressure was significantly higher with ketamine/ propofol admixture sedation as compared to nonketamine/ propofol admixture sedation when induction of anesthesia is required for airway manipulation. Although not statistically significant, heart rate, diastolic blood pressure, and MAP were higher when ketamine/propofol admixture-based sedation was employed versus nonketamine/propofol admixture based-sedation for induction of anesthesia. Furthermore, one study indicated a possible beneficial effect on pain scores in the immediate 24 hours postdrug administration [27].

Evidence has demonstrated that maintaining hemodynamics around the time of airway instrumentation such as endotracheal intubation is vitally important, in both the critically ill and noncritically ill. For example, in the critically ill, peri-intubation hypotension was associated with increased odds of dying (39\% peri-intubation hypotension versus $30 \%$ no peri-intubation hypotension, $P=0.045$ ) and increased odds of experiencing intensive care unit length of stay greater than 14 days, duration of mechanical ventilation longer than 7 days, and requiring renal replacement therapy (odds ratio 2.0, 95\% CI: 1.30-3.07, $P=0.0017$ ) [1]. These 


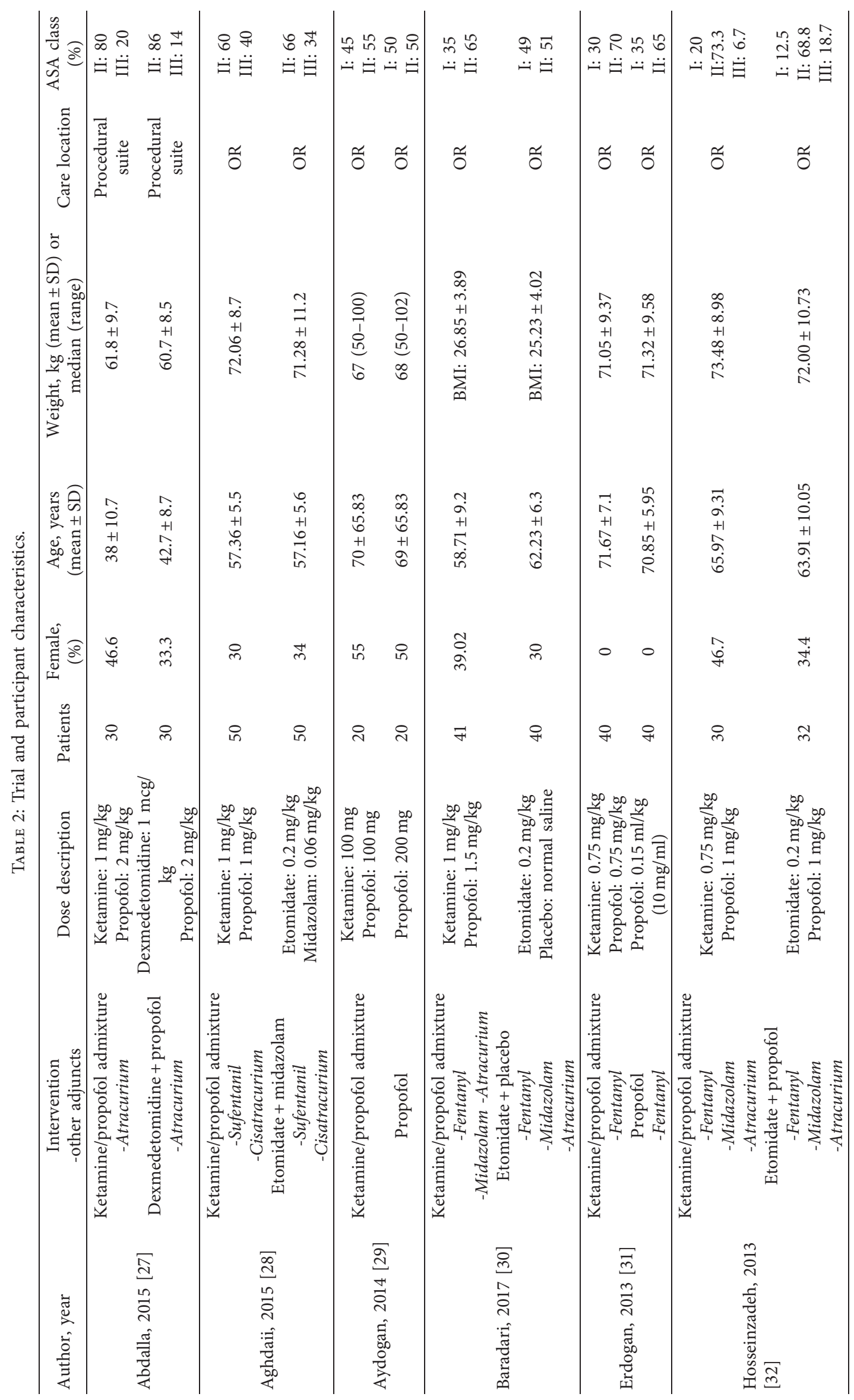




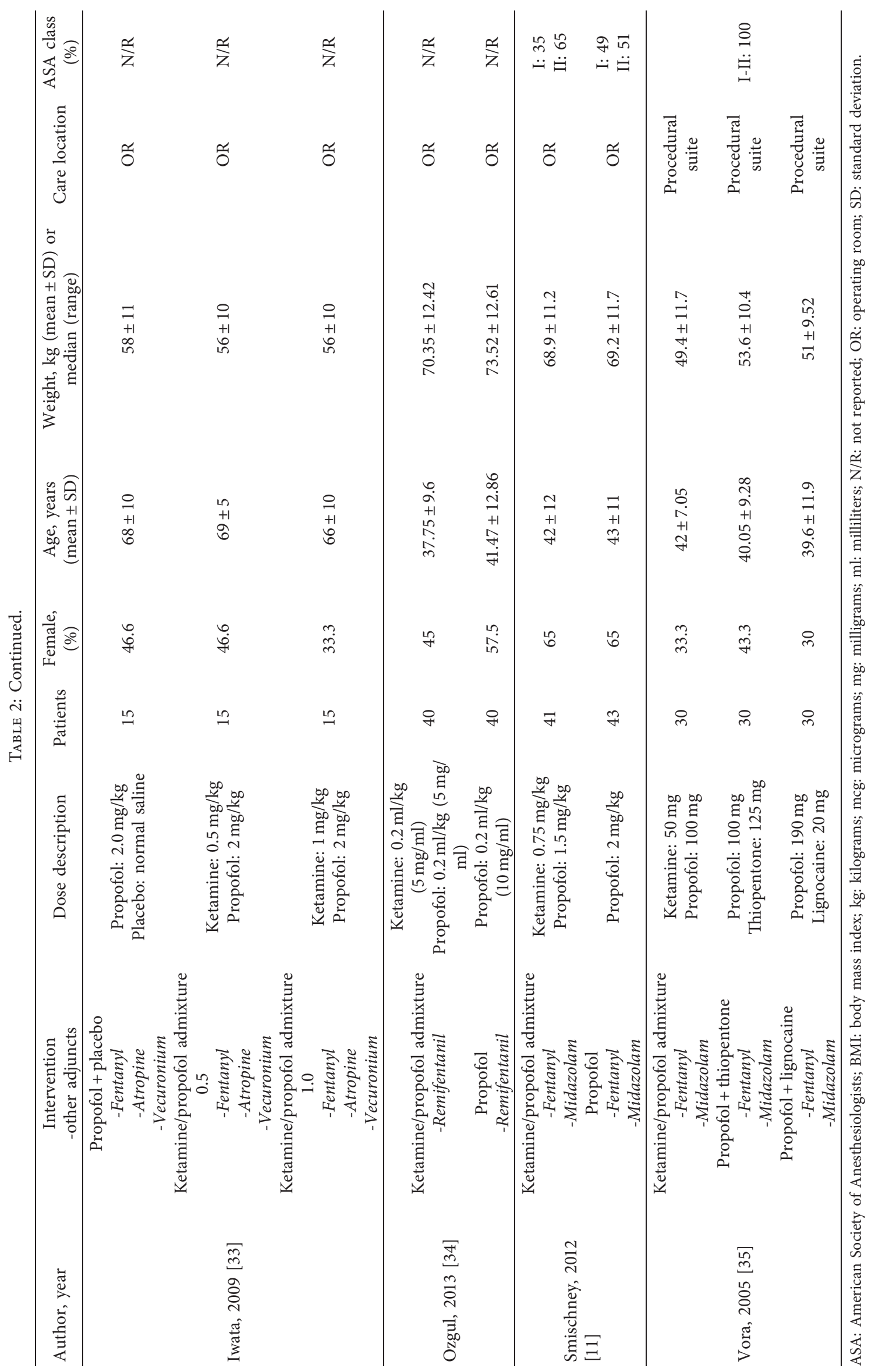


TABLE 3: Risk of bias.

\begin{tabular}{|c|c|c|c|c|c|c|c|c|}
\hline Trial & $\begin{array}{l}\text { Random } \\
\text { sequence } \\
\text { generation }\end{array}$ & $\begin{array}{l}\text { Allocation } \\
\text { concealment }\end{array}$ & $\begin{array}{c}\text { Participant/ } \\
\text { personnel } \\
\text { blinding }\end{array}$ & $\begin{array}{l}\text { Blinding of } \\
\text { outcome } \\
\text { assessment }\end{array}$ & $\begin{array}{c}\text { Incomplete } \\
\text { outcome data, \% } \\
\text { loss to follow-up }\end{array}$ & $\begin{array}{l}\text { Selective } \\
\text { reporting }\end{array}$ & $\begin{array}{c}\text { Other } \\
\text { sources of } \\
\text { bias }\end{array}$ & $\begin{array}{l}\text { Overall } \\
\text { risk }\end{array}$ \\
\hline Abdalla, 2015 & Low risk & Unclear & Low risk & Unclear & Low risk, $0 \%$ & Unclear & Unclear & High \\
\hline Aghdaii, 2015 & Low risk & Unclear & Unclear & Low risk & Unclear, N/R & Unclear & Unclear & High \\
\hline Aydogan, 2014 & Low risk & Low risk & Low risk & Low risk & Low risk, $0 \%$ & Unclear & Low risk & Low \\
\hline Baradari, 2017 & High risk & Low risk & Low risk & Low risk & Low risk, $3.5 \%$ & Low risk & Low risk & Low \\
\hline Erdogan, 2013 & Low risk & Unclear & Low risk & Unclear & Low risk, $0 \%$ & Unclear & Unclear & High \\
\hline $\begin{array}{l}\text { Hosseinzadeh, } \\
2013\end{array}$ & Low risk & Unclear & Low risk & Low risk & Unclear, N/R & Unclear & Low risk & Moderate \\
\hline Iwata, 2009 & Low risk & Unclear & Unclear & Low risk & Unclear, N/R & Unclear & Unclear & High \\
\hline Ozgul, 2013 & Low risk & Unclear & Low risk & Unclear & Unclear, N/R & Unclear & Unclear & High \\
\hline $\begin{array}{l}\text { Smischney, } \\
2012\end{array}$ & Low risk & Low risk & Low risk & Low risk & Low risk, $0 \%$ & Unclear & Low risk & Low \\
\hline Vora, 2005 & Unclear & Unclear & Low risk & Unclear & Unclear, N/R & High risk & Unclear & High \\
\hline
\end{tabular}

N/R: not reported.

TABLE 4: Hemodynamic outcomes.

\begin{tabular}{|c|c|c|c|c|}
\hline Intervention & Outcome & Conclusion & $\begin{array}{l}\text { Study design/sample } \\
\text { size }\end{array}$ & Certainty of evidence \\
\hline $\begin{array}{l}\text { Ketamine/propofol admixture vs. } \\
\text { nonketamine/propofol admixture }\end{array}$ & $\begin{array}{l}\text { HR } 10 \text { min } \\
\text { SBP } 5 \text { min } \\
\text { SBP } \\
10 \mathrm{~min} \\
\text { DBP } \\
5 \mathrm{~min} \\
\text { DBP } \\
10 \mathrm{~min} \\
\mathrm{MAP} \\
5 \mathrm{~min} \\
\mathrm{MAP} \\
10 \mathrm{~min}\end{array}$ & $\begin{array}{c}\text { WMD, } 3.36 \mathrm{mmHg}(95 \% \mathrm{CI} \text {, } \\
-0.88 \text { to } 7.60), I^{2}=88.6 \% \\
\text { WMD, } 0.36 \mathrm{mmHg}(95 \% \mathrm{CI} \text {, } \\
-2.57 \text { to } 3.29), I^{2}=69.9 \% \\
\text { WMD, } 9.67 \mathrm{mmHg}(95 \% \mathrm{CI} \text {, } \\
1.48 \text { to } 17.86), I^{2}=87.2 \% \\
\text { WMD, } 4.56 \mathrm{mmHg}(95 \% \mathrm{CI} \text {, } \\
-1.09 \text { to } 10.20), I^{2}=0.0 \% \\
\text { WMD, } 2.18 \mathrm{mmHg}(95 \% \mathrm{CI} \text {, } \\
-2.82 \text { to } 7.19), I^{2}=73.1 \% \\
\text { WMD, } 4.80 \mathrm{mmHg}(95 \% \mathrm{CI} \text {, } \\
0.24 \text { to } 9.36), I^{2}=\mathrm{N} / \mathrm{A} \\
\text { WMD, } 3.28 \mathrm{mmHg}(95 \% \mathrm{CI} \text {, } \\
-0.94 \text { to } 7.49), I^{2}=69.9 \% \\
\text { WMD, } 4.08 \mathrm{mmHg}(95 \% \mathrm{CI} \text {, } \\
-0.22 \text { to } 8.39), I^{2}=41.4\end{array}$ & $\begin{array}{c}\text { Six RCTs }(n=465) \\
11,27-31 \\
\text { Three RCTs }(n=244) \\
11,27,29 \\
\text { Five RCTs }(n=385) \\
11,27-28,30-31 \\
\text { Two RCTs }(n=164) \\
11,27 \\
\text { Four RCTs }(n=305) \\
11,28,30-31 \\
\text { One RCT }(n=84) 11 \\
\text { Four RCTs }(n=345) \\
11,29-31 \\
\text { Two RCTs }(n=164) \\
11,29\end{array}$ & $\begin{array}{l}\text { Very low due to risk of bias, } \\
\text { imprecision, and inconsistency } \\
\text { Very low due to risk of bias, } \\
\text { imprecision, and inconsistency } \\
\text { Low due to risk of bias and } \\
\text { inconsistency } \\
\text { Low due to risk of bias and } \\
\text { imprecision } \\
\text { Very low due to risk of bias, } \\
\text { inconsistency, and imprecision } \\
\text { Low due to risk of bias and } \\
\text { imprecision } \\
\text { Very low due to risk of bias, } \\
\text { imprecision, and inconsistency } \\
\text { Low due to risk of bias and } \\
\text { imprecision }\end{array}$ \\
\hline
\end{tabular}

CI: confidence interval; HR: heart rate; WMD: weighted mean difference; RCT: randomized controlled trial; SBP: systolic blood pressure; DBP: diastolic blood pressure; MAP: mean arterial pressure; N/A: not applicable.

observations have been demonstrated in other populations of critically ill patients [36]. In noncritically ill patients undergoing noncardiac surgery, a significant fraction of all hypotensive events occurred before skin incision and, therefore, due to anesthetic management as demonstrated in one study. In addition, this study revealed that the odds of developing acute kidney injury increased with MAP less than $65 \mathrm{mmHg}$ (odds ratio 1.02, 95\% CI: 1.01-1.04, $P=0.004$ ) [37]. Moreover, patients undergoing noncardiac surgery who experienced intraoperative hypotension defined as MAP less than $65 \mathrm{mmHg}$ or a MAP decrease of $20 \%$ from baseline had increased odds of developing both myocardial and kidney injury [38]. Likewise, patients who developed greater than a 30\% decrease in MAP from baseline intraoperatively had increased odds of developing postoperative stroke (odds ratio 1.013/min hypotension, 99.9\% CI: $1.000-1.025, P \leq 0.001)$ [39].

A recent systematic review suggested poor outcomes with increased end-organ injury in patients undergoing noncardiac surgery who experience MAP decreases $<80 \mathrm{~mm}$ $\mathrm{Hg}$ for $\geq 10$ minutes [40]. By the same token, elevated MAP thresholds have been postulated to be beneficial in the critically ill population. For example, one study demonstrated improved microcirculation in septic shock patients with previous hypertension when MAP was increased above a threshold of $65 \mathrm{mmHg}$ [41]. Furthermore, others have shown that maintaining MAP well above $65 \mathrm{mmHg}$ (i.e., $85 \mathrm{mmHg}$ ) in septic, critically ill patients may result in less myocardial and kidney injury as well as decreasing overall mortality [42].

Given that periprocedure (i.e., endotracheal intubation) hemodynamics are influenced by sedation and the preponderance of evidence demonstrating that preventing hypotensive episodes, especially with MAPs less than $65 \mathrm{mmHg}$ or SBPs less than $90 \mathrm{mmHg}$, and maintaining perfusion pressure near baseline improves outcomes, the selection of an intravenous anesthetic agent during a procedure (i.e., endotracheal intubation) ought to have this goal 


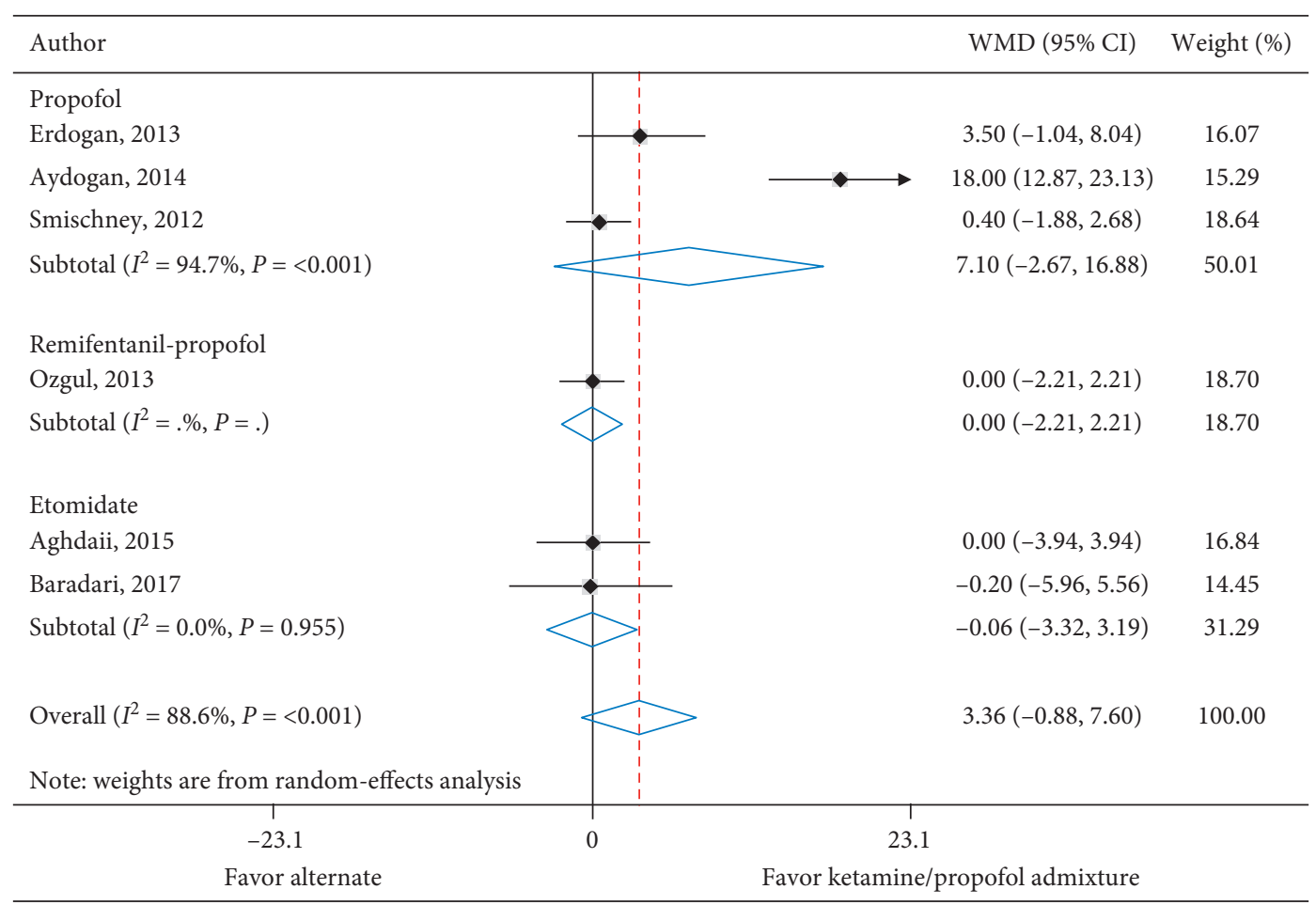

CI: confidence interval; WMD: weighted mean difference

(a)

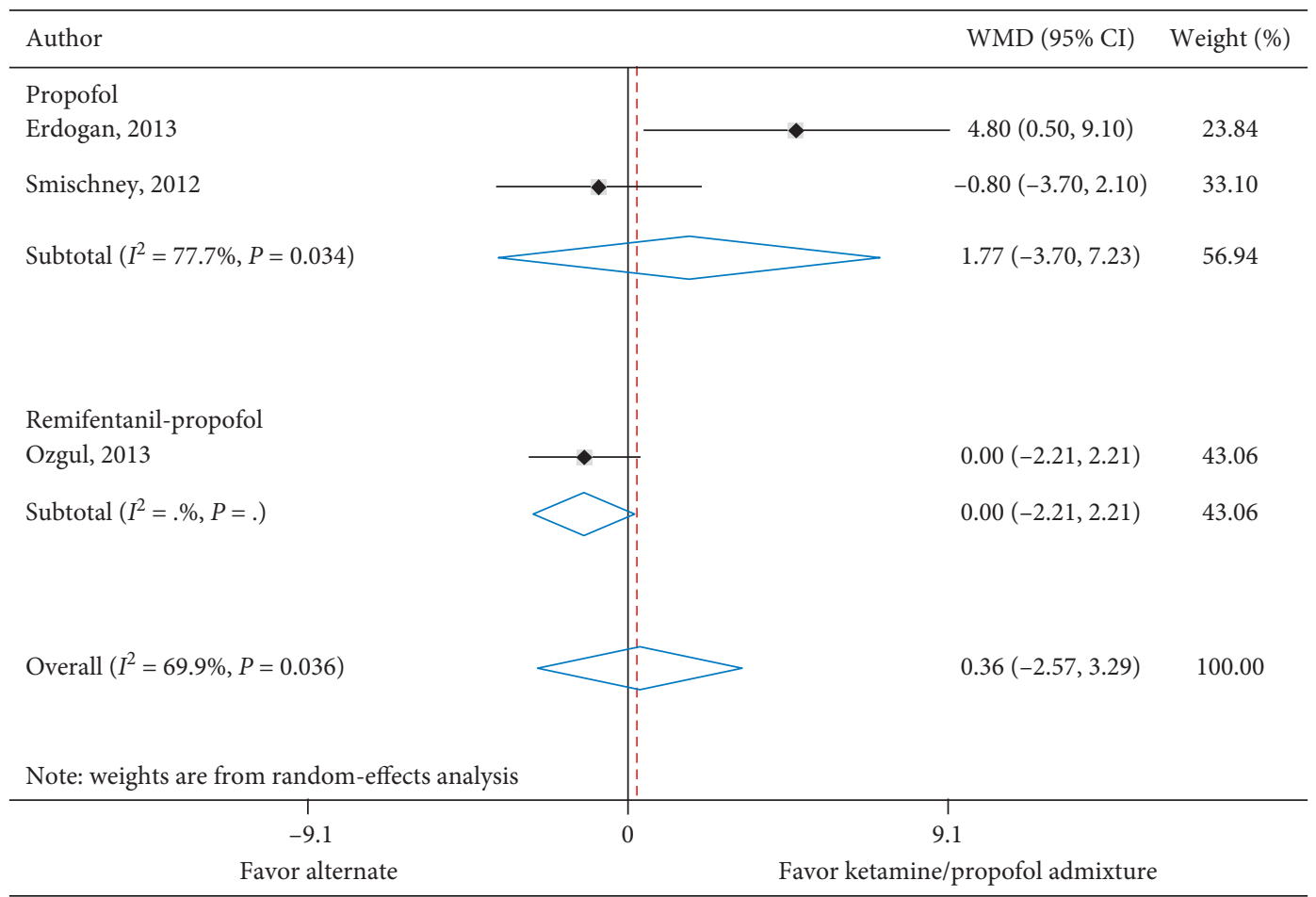

CI: confidence interval; WMD: weighted mean difference

(b)

Figure 2: (a) Heart rate at 5 minutes. (b) Heart rate at 10 minutes.

in mind. To this end, a recent editorial questioned the current blood pressure parameter of MAP $65 \mathrm{mmHg}$ in the intensive care unit, which largely comes from two retrospective studies [43]. The authors suggested that perhaps mean perfusion pressure, which is defined by MAP minus central venous pressure, may be a better parameter to 


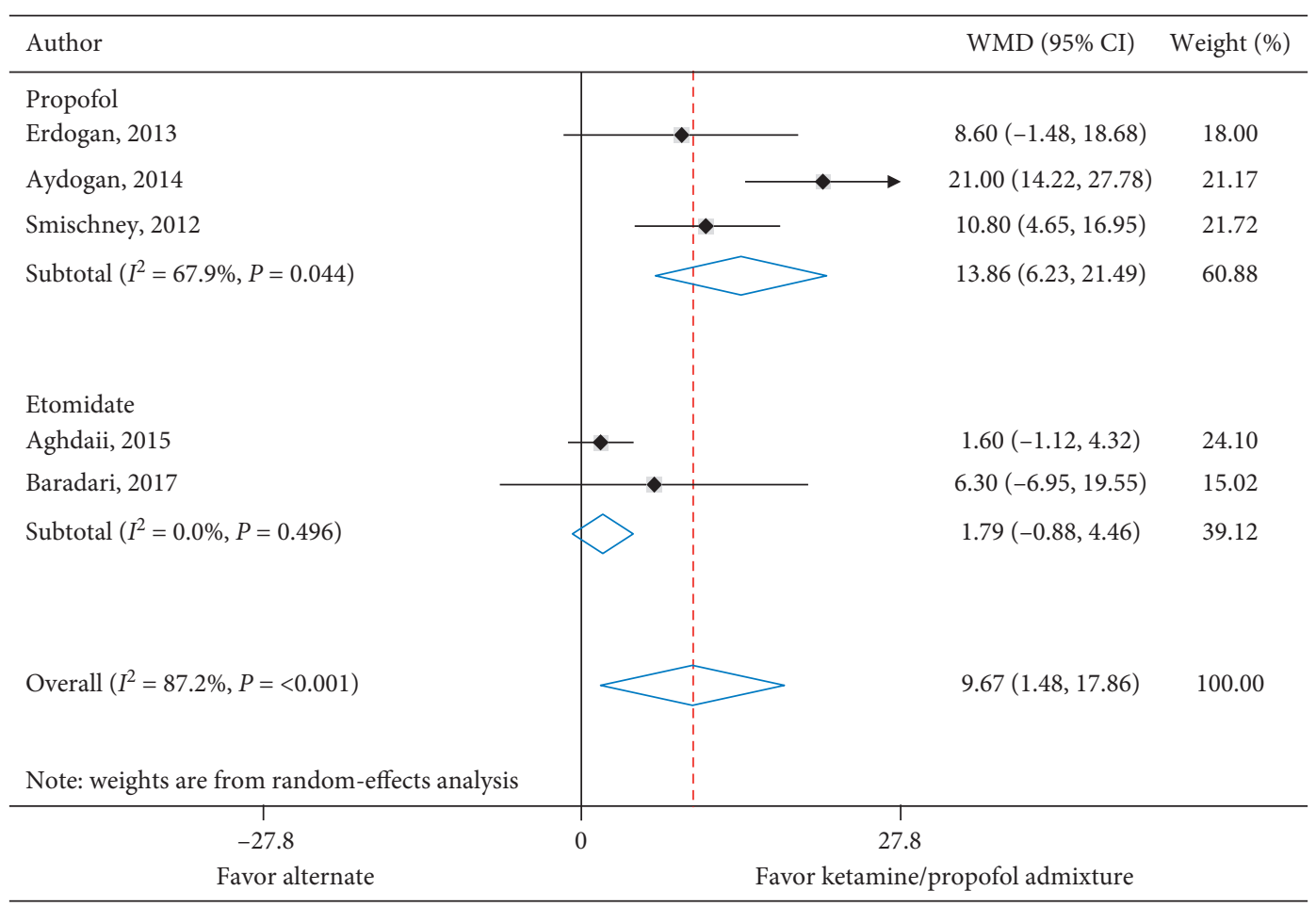

CI: confidence interval; WMD: weighted mean difference

(a)

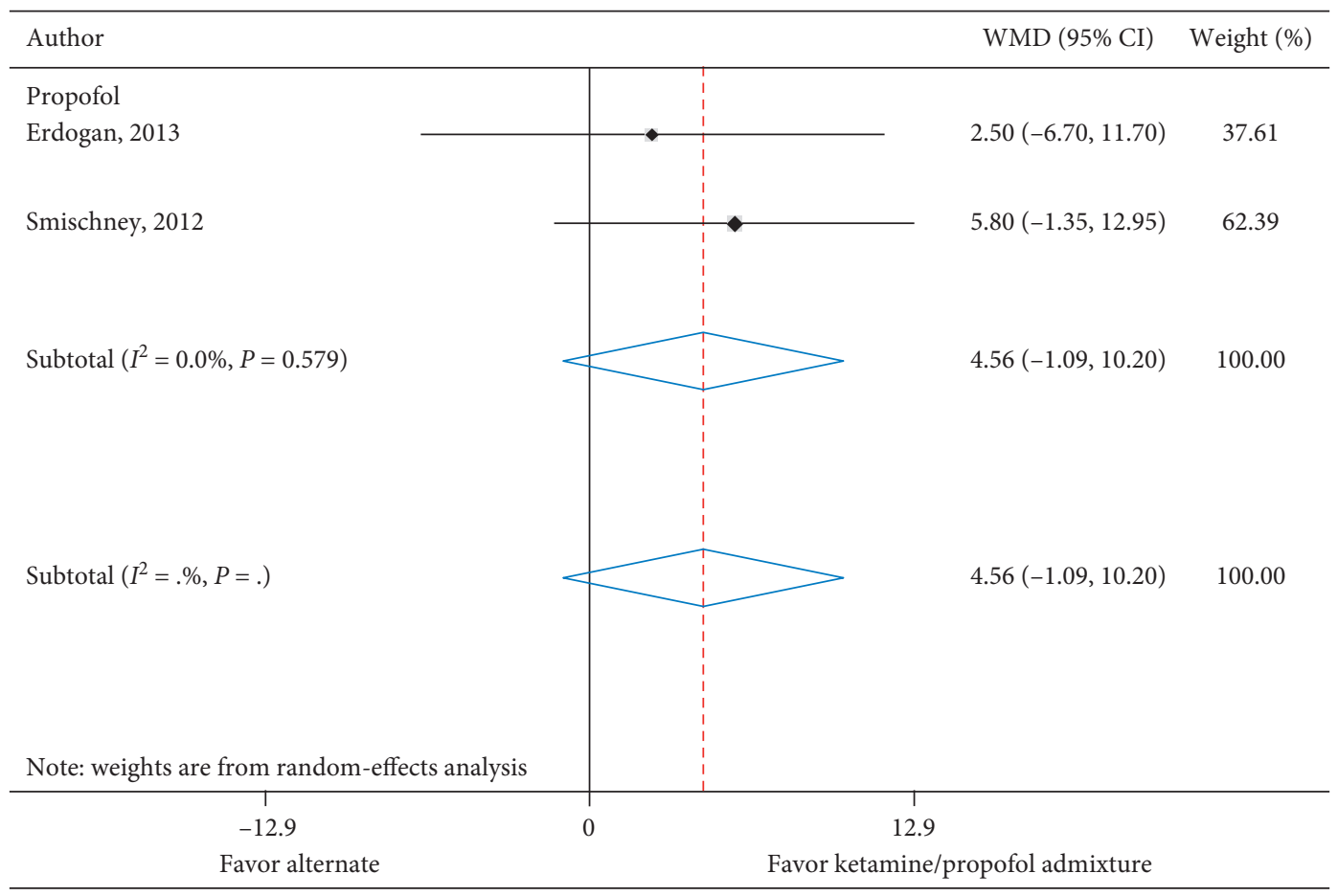

CI: confidence interval; WMD: weighted mean difference

(b)

FIGURE 3: (a) Systolic blood pressure at 5 minutes. (b) Systolic blood pressure at 10 minutes.

use as compared to the current parameter of MAP. While we did not look at long-term outcomes in our current study, we demonstrated that induction with ketamine/propofol admixture rather than other intravenous anesthetic agents resulted in better hemodynamics. Therefore, it is possible that this may translate into improved patient-centered outcomes. Although elevated perfusion pressures may be advantageous in the general sense, this has to be individually 


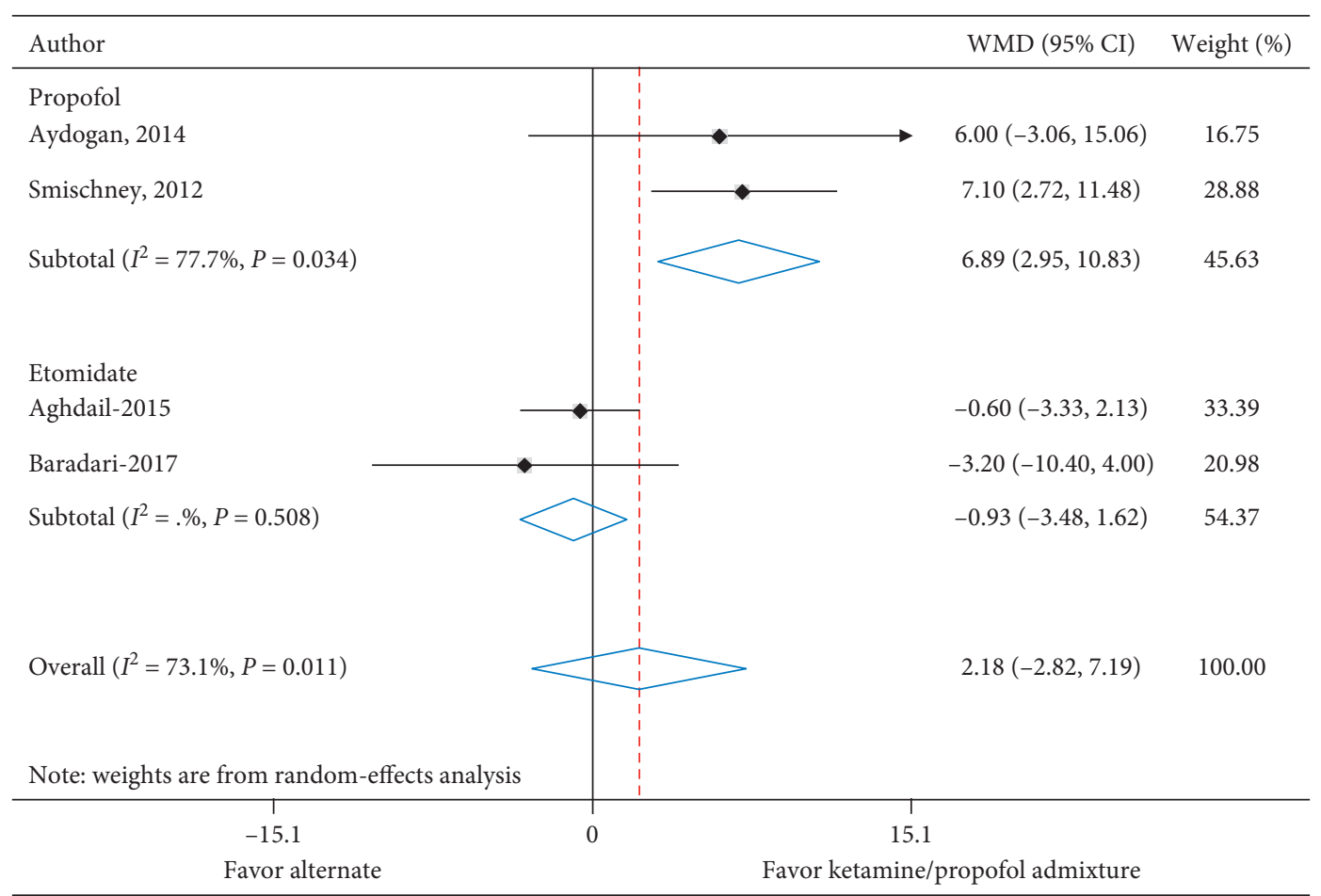

CI: confidence interval; WMD: weighted mean difference

(a)

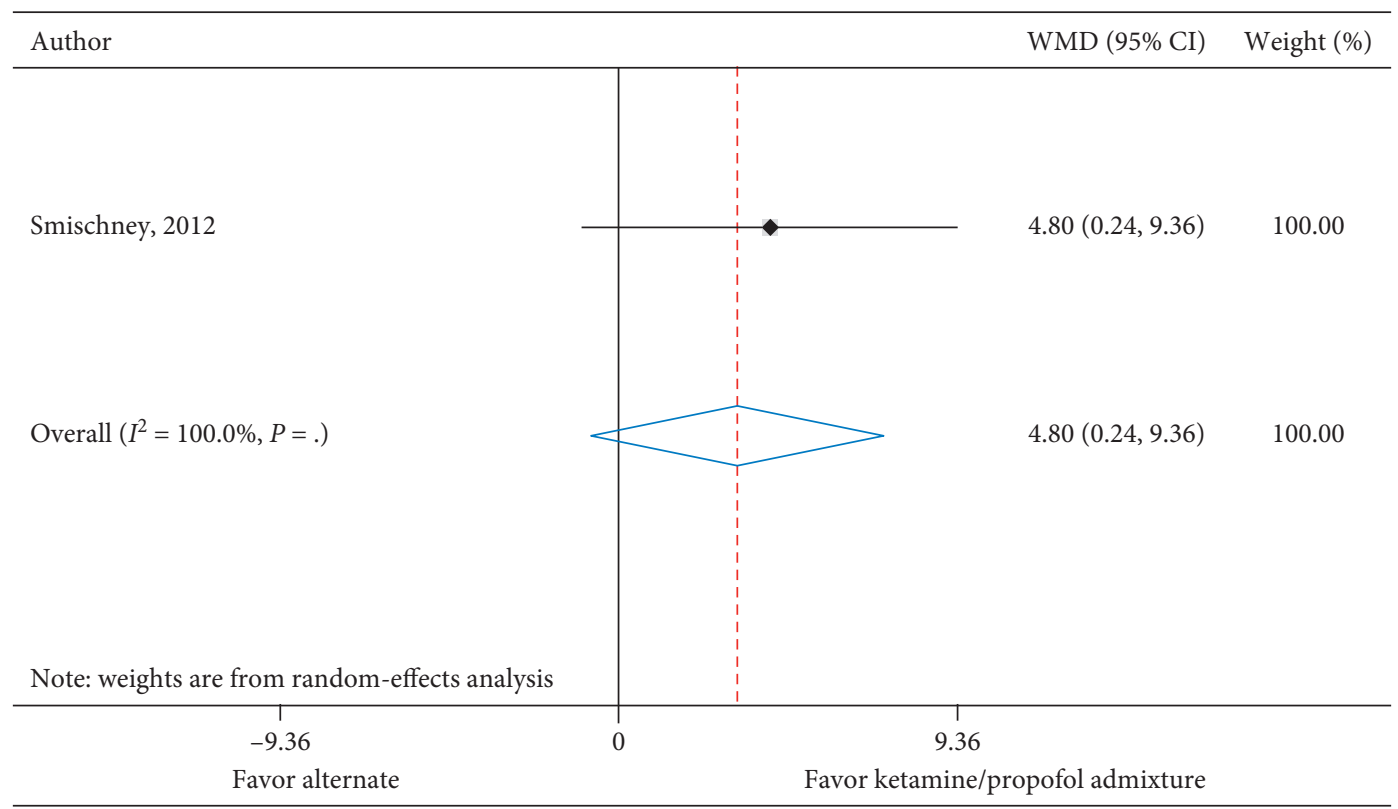

CI: confidence interval; WMD: weighted mean difference

(b)

Figure 4: (a) Diastolic blood pressure at 5 minutes. (b) Diastolic blood pressure at 10 minutes.

tailored to each patient as high pressure/heart rate may be harmful to some. For example, ketamine may exacerbate myocardial ischemia in noncompensated coronary artery disease or hypertension through its effects on central nervous system stimulation and inhibition of norepinephrine reuptake (i.e., cardiac population) [10].
The current systematic review and meta-analysis are consistent with prior reviews of ketamine/propofol admixture administration for procedural sedation. Prior reviews have demonstrated better hemodynamics with ketamine/propofol admixture-based procedural sedation than with nonketamine/propofol admixture-based 


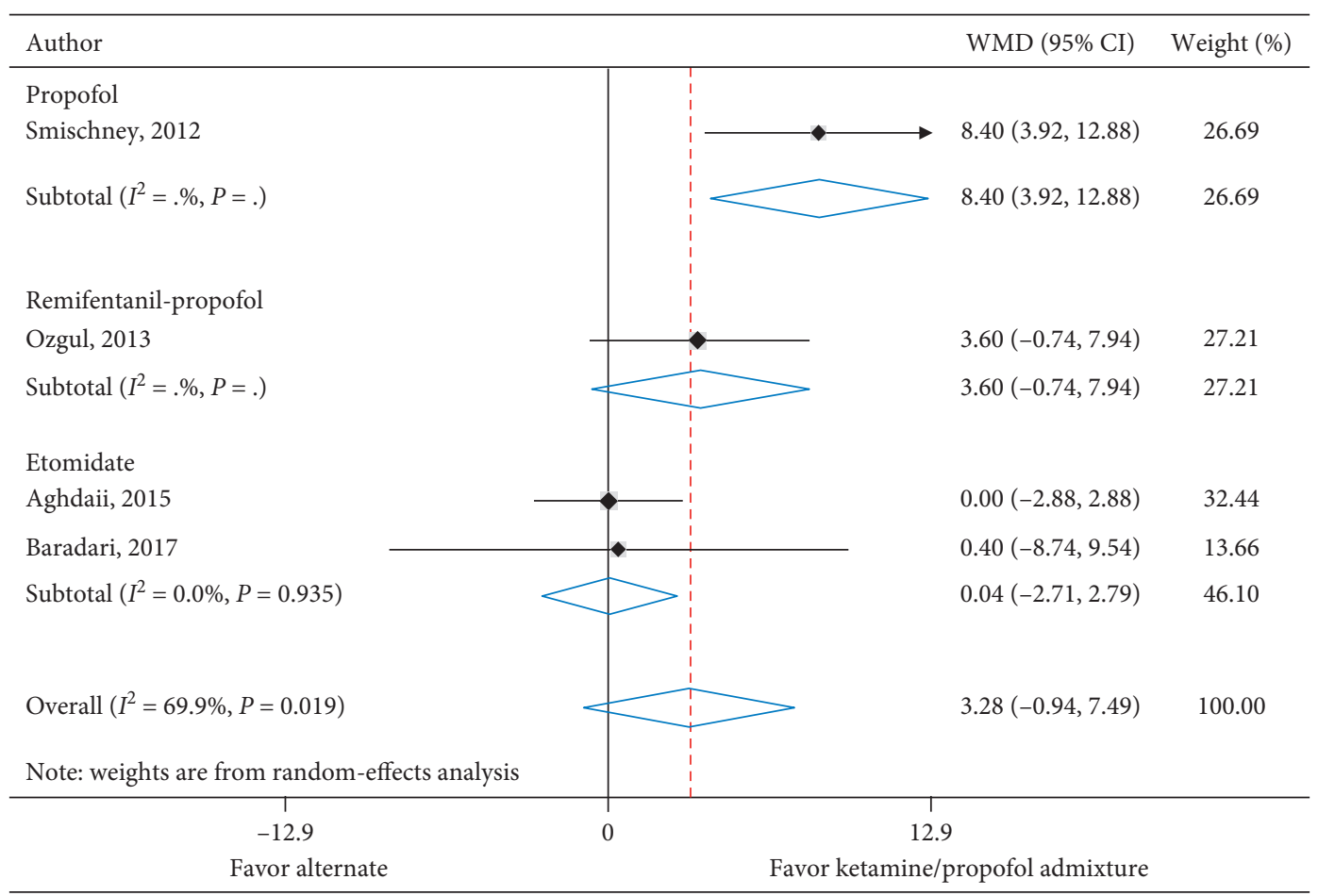

CI: confidence interval; WMD: weighted mean difference

(a)

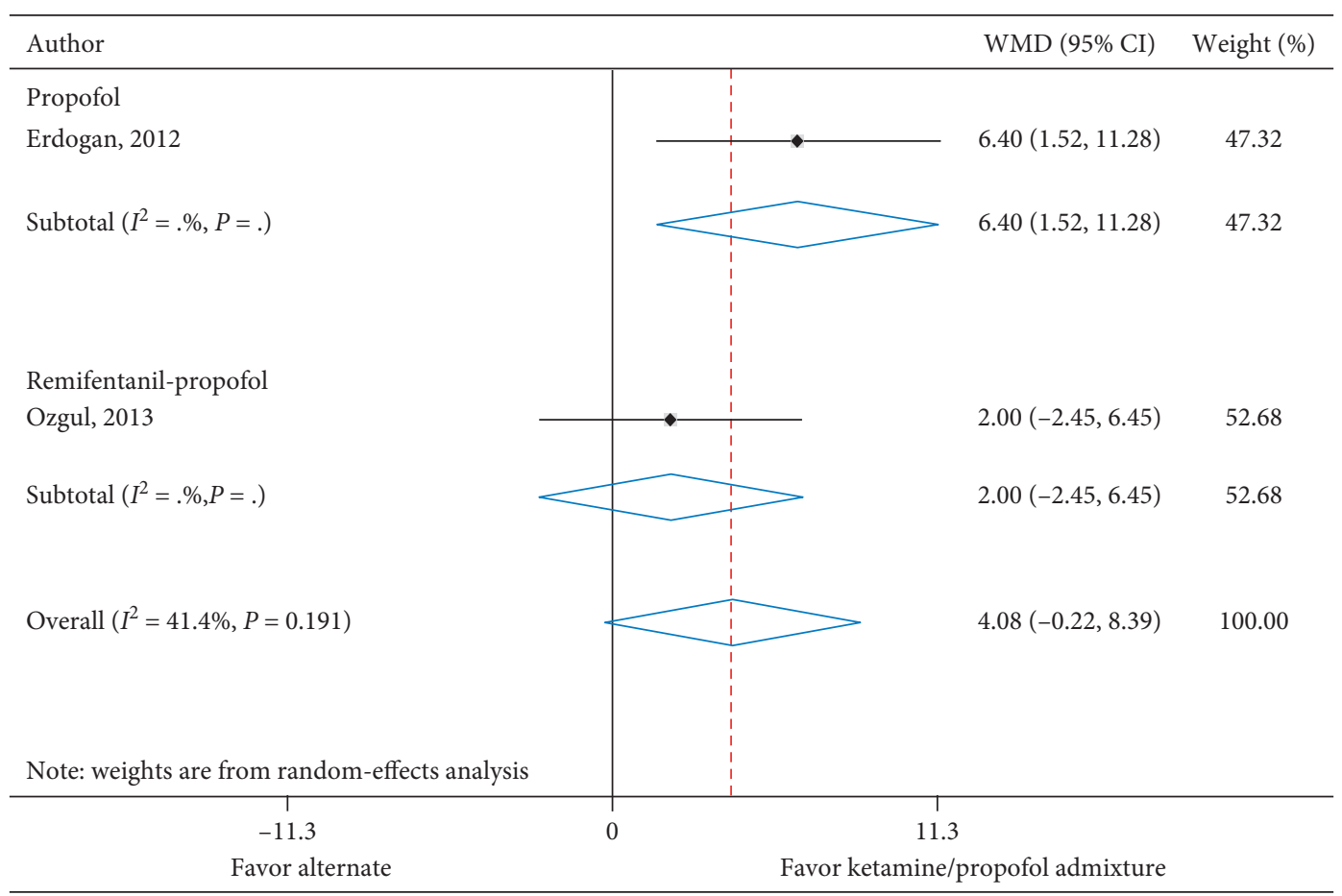

CI: confidence interval; WMD: weighted mean difference

(b)

Figure 5: (a) Mean arterial pressure at 5 minutes. (b) Mean arterial pressure at 10 minutes.

procedural sedation. For example, a recent review demonstrated that ketamine/propofol admixture-based procedural sedation was effective in reducing cardiovascular complications (relative risk for hypotension 0.11, 95\% CI:
$0.17-0.97, P=0.04$; relative risk for bradycardia $0.47,95 \%$ CI: $0.28-0.72, P=0.008)$. The authors also demonstrated similar rates of psychotomimetic complications and nauseavomiting when compared to propofol [18]. A second review 
assessed adverse respiratory events and recovery times with ketamine/propofol admixture-based procedural sedation as compared to propofol-based procedural sedation. The authors noted that adverse respiratory events were significantly reduced with ketamine/propofol admixture as compared to propofol (risk ratio 0.82, 95\% CI: 0.68-0.99, $P=0.04$ ); however, recovery times were similar in both groups. Hemodynamic thresholds were not assessed in this review [44]. Although the above reviews point towards the safety of this combination, the admixture can, by its very nature, lead to some undesired effects. Apart from the use of ketamine in the cardiac population (discussed above), ketamine causes initial release of glutamate through nicotinamide adenine dinucleotide phosphate hydrogen oxidase 2 leading to emergence delirium with eventual blockade of glutamate's effects through N-methyl-D-aspartate antagonism. Emergence delirium could be devastating to patients with underlying mental illnesses such as schizophrenia, bipolar disorder, or posttraumatic stress disorder to name a few. This emergence delirium is dose dependent and also affected by cointerventions such as benzodiazepine administration. Interestingly, propofol has been shown to blunt glutamate's

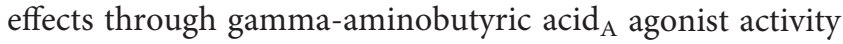
[10]. This may explain the findings in the literature demonstrating a reduced rate of emergence delirium with the admixture $(\sim 30 \%$ with ketamine only vs. $<5 \%)$ [10-12, 14, 31].

Our study has several benefits. First, we focused on a theoretical advantage of ketamine/propofol admixture over other agents used for induction of anesthesia during procedures such as endotracheal intubation, namely, hemodynamic preservation. Furthermore, we extracted hemodynamic data in the immediate period after drug administration, thereby limiting the effects of other cointerventions. Second, we extracted data on all drug comparisons to ketamine/propofol admixture. Third, we included trials that had either similar or near equivalent dosing of ketamine/propofol admixture.

4.1. Limitations. Similar to other systematic reviews and meta-analyses, our results are limited by clinical trial quality. Although our search strategy was comprehensive, we included only English-language articles and published articles after 2000 and thus may have missed trials not published in English or published before the year 2000. Hemodynamic data were not recorded similarly in all trials nor was the frequency of hemodynamic data collected. Furthermore, 5 and 10 minutes may be an oversimplification of hemodynamic representation as intravenous anesthetics used for induction of anesthesia have a short initial elimination halflife and, therefore, collecting hemodynamic data every minute would have been ideal [10]. Moreover, we did not calculate MAP from trials reporting systolic and diastolic blood pressure because individual patient data were not reported. Three studies met our inclusion criteria and were included in the systematic review. However, we did not include these studies in the meta-analysis as we were unable to extract measures of variation [32, 33, 35]. Data on vasoactive medications which could alter hemodynamics and affect our estimates were not reported in the majority of included trials. We do not report subgroup meta-analyses for the different patient populations or interventions studied due to the limited number of studies in each meta-analysis (only 1 meta-analysis included 3 studies and the remainder had 2 studies). Thus, any subgroup and sensitivity analyses would reduce the number further with only 1 study, which is already presented well in the forest plots. Lastly, our systematic review illustrated moderate to high heterogeneity in the analyses and high risk of bias of the included studies. With such small numbers of studies in the meta-analysis, advanced bias evaluations were not appropriate. Thus, our results should be interpreted with caution.

\section{Conclusions}

We demonstrated a potentially better hemodynamic profile with ketamine/propofol admixture-based induction of anesthesia versus nonketamine/propofol admixture-based induction of anesthesia for procedures requiring airway manipulation such as endotracheal intubation. However, given moderate to high heterogeneity of trials included in the current meta-analysis, our results should be interpreted with caution.

\section{Conflicts of Interest}

The authors declare that they have no conflicts of interest.

\section{Acknowledgments}

This work was supported by funding from the Department of Anesthesiology and Perioperative Medicine and the Critical Care Independent Multidisciplinary Practice at Mayo Clinic Rochester, Minnesota.

\section{References}

[1] R. S. Green, A. F. Turgeon, L. A. McIntyre et al., "Postintubation hypotension in intensive care unit patients: a multicenter cohort study," Journal of Critical Care, vol. 30, no. 5, pp. 1055-1060, 2015.

[2] N. J. Smischney, O. Demirci, D. A. Diedrich et al., "Incidence of and risk factors for post-intubation hypotension in the critically ill," Medical Science Monitor, vol. 22, pp. 346-355, 2016.

[3] A. C. Heffner, D. S. Swords, M. L. Nussbaum, J. A. Kline, and A. E. Jones, "Predictors of the complication of postintubation hypotension during emergency airway management," Journal of Critical Care, vol. 27, no. 6, pp. 587-593, 2012.

[4] T. G. Monk, M. R. Bronsert, W. G. Henderson et al., “Association between intraoperative hypotension and hypertension and 30-day postoperative mortality in noncardiac surgery," Anesthesiology, vol. 123, no. 2, pp. 307-319, 2015.

[5] R. S. Green and M. B. Butler, "Postintubation hypotension in general anesthesia," Journal of Intensive Care Medicine, vol. 31, no. 10, pp. 667-675, 2016.

[6] J. B. Bijker, W. A. van Klei, T. H. Kappen, L. van Wolfswinkel, K. G. M. Moons, and C. J. Kalkman, "Incidence of intraoperative hypotension as a function of the chosen definition," Anesthesiology, vol. 107, no. 2, pp. 213-220, 2007. 
[7] R. Green, B. Hutton, J. Lorette, D. Bleskie, L. Mclntyre, and D. Fergusson, "Incidence of postintubation hemodynamic instability associated with emergent intubations performed outside the operating room: a systematic review," CJEM, vol. 16, no. 1, pp. 69-79, 2014.

[8] N. J. Smischney, M. O. Seisa, K. J. Heise et al., "Predictors of hemodynamic derangement during intubation in the critically ill: a nested case-control study of hemodynamic managementPart II," Journal of Critical Care, vol. 44, pp. 179-184, 2018.

[9] N. J. Smischney, M. O. Seisa, J. Cambest et al., "The incidence of and risk factors for postintubation hypotension in the immunocompromised critically ill adult," Journal of Intensive Care Medicine, vol. 34, no. 7, pp. 578-586, 2019.

[10] R. D. Miller, Miller's Anesthesia, Elsevier, Philadelphia, PA, USA, 8th edition, 2014.

[11] N. J. Smischney, M. L. Beach, R. W. Loftus, T. M. Dodds, and M. D. Koff, "Ketamine/propofol admixture (ketofol) is associated with improved hemodynamics as an induction agent," Journal of Trauma and Acute Care Surgery, vol. 73, no. 1, pp. 94-101, 2012.

[12] G. Yousef and K. Elsayed, "A clinical comparison of ketofol (ketamine and propofol admixture) versus propofol as an induction agent on quality of laryngeal mask airway insertion and hemodynamic stability in children," Anesthesia: Essays and Researches, vol. 7, no. 2, pp. 194-199, 2013.

[13] A. Gallo de Moraes, C. J. Racedo Africano, S. S. Hoskote et al., "Ketamine and propofol combination ("ketofol") for endotracheal intubations in critically ill patients: a case series," American Journal of Case Reports, vol. 16, pp. 81-86, 2015.

[14] E. V. Willman and G. Andolfatto, "A prospective evaluation of "ketofol" (Ketamine/Propofol combination) for procedural sedation and analgesia in the emergency department," Annals of Emergency Medicine, vol. 49, no. 1, pp. 23-30, 2007.

[15] A. Nejati, R. S. Moharari, H. Ashraf, A. Labaf, and K. Golshani, "Ketamine/propofol versus midazolam/fentanyl for procedural sedation and analgesia in the emergency department: a randomized, prospective, double-blind trial," Academic Emergency Medicine, vol. 18, no. 8, pp. 800-806, 2011.

[16] G. Andolfatto and E. Willman, "A prospective case series of single-syringe ketamine-propofol (ketofol) for emergency department procedural sedation and analgesia in adults," Academic Emergency Medicine, vol. 18, no. 3, pp. 237-245, 2011.

[17] S. Arora, "Combining ketamine and propofol ("ketofol") for emergency department procedural sedation and analgesia: a review," The Western Journal of Emergency Medicine, vol. 9, no. 1, pp. 20-23, 2008.

[18] M. Jalili, M. Bahreini, A. Doosti-Irani, R. Masoomi, M. Arbab, and H. Mirfazaelian, "Ketamine-propofol combination (ketofol) vs propofol for procedural sedation and analgesia: systematic review and meta-analysis," The American Journal of Emergency Medicine, vol. 34, no. 3, pp. 558-569, 2016.

[19] D. Moher, L. Shamseer, M. Clarke et al., "Preferred reporting items for systematic review and meta-analysis protocols (PRISMA-P) 2015 statement," Systematic Reviews, vol. 4, no. 1, p. 1, 2015.

[20] L. Shamseer, D. Moher, M. Clarke et al., "Preferred reporting items for systematic review and meta-analysis protocols (PRISMA-P) 2015: elaboration and explanation," BMJ, vol. 349, no. jan02 1, p. g7647, 2015.

[21] J. Eden, L. Levit, A. Berg, and S. Morton, Finding what Works in Health Care: Standards for Systematic Reviews, The National Academies Press, Washington, DC, USA, 2011.
[22] S. S.-L. Wong, N. L. Wilczynski, and R. B. Haynes, "Developing optimal search strategies for detecting clinically sound treatment studies in EMBASE," The Journal of the Medical Library Association, vol. 94, no. 1, pp. 41-47, 2006.

[23] M. H. Murad, "Clinical Practice guidelines," Mayo Clinic Proceedings, vol. 92, no. 3, pp. 423-433, 2017.

[24] M. Woodward, Epidemiology: Study Design and Data Analysis, Chapman \& Hall/CRC, New York, NY, USA, 2nd edition, 2004.

[25] J. P. T. Higgins and S. G. Thompson, "Quantifying heterogeneity in a meta-analysis," Statistics in Medicine, vol. 21, no. 11, pp. 1539-1558, 2002.

[26] J. P. T. Higgins, S. G. Thompson, J. J. Deeks, and D. G. Altman, "Measuring inconsistency in meta-analyses," BMJ, vol. 327, no. 7414, pp. 557-560, 2003.

[27] M. W. Abdalla, S. M. El Shal, A. I. El Sombaty, N. M. Abdalla, and R. B. Zeedan, "Propofol dexmedetomidine versus propofol ketamine for anesthesia of endoscopic retrograde cholangiopancreatography (ERCP) (A randomized comparative study)," Egyptian Journal of Anaesthesia, vol. 31, no. 2, pp. 97-105, 2015.

[28] N. Aghdaii, M. Ziyaeifard, S. Z. Faritus, and R. Azarfarin, "Hemodynamic responses to two different anesthesia regimens in compromised left ventricular function patients undergoing coronary artery bypass graft surgery: etomidatemidazolam versus propofol-ketamine," Anesthesiology and Pain Medicine, vol. 5, no. 3, Article ID e27966, 2015.

[29] M. S. Aydogan, S. Demirel, M. A. Erdogan, P. Firat, C. Colak, and M. Durmus, "Effects of ketamine-propofol mixture on intraocular pressure and hemodynamic in elderly patients: a randomized double-blind trial," Turkish Journal of Anesthesia and Reanimation, vol. 42, no. 1, pp. 12-18, 2014

[30] A. G. Baradari, A. Alipour, M. R. Habibi, S. Rashidaei, and A. E. Zeydi, "A randomized clinical trial comparing hemodynamic responses to ketamine-propofol combination (ketofol) versus etomidate during anesthesia induction in patients with left ventricular dysfunction undergoing coronary artery bypass graft surgery," Archives of Medical Science, vol. 5, no. 5, pp. 1102-1110, 2017.

[31] M. A. Erdogan, Z. Begec, M. S. Aydogan et al., "Comparison of effects of propofol and ketamine-propofol mixture (ketofol) on laryngeal mask airway insertion conditions and hemodynamics in elderly patients: a randomized, prospective, double-blind trial," Journal of Anesthesia, vol. 27, no. 1, pp. 12-17, 2013.

[32] H. Hosseinzadeh, M. Eidy, S. E. Golzari, and M. Vasebi, "Hemodynamic stability during induction of anesthesia in elderly patients: propofol + ketamine versus propofol + etomidate," Journal of Cardiovascular and Thoracic Research, vol. 5, no. 2, pp. 51-54, 2013.

[33] M. Iwata, S. Inoue, M. Kawaguchi et al., "Ketamine eliminates propofol pain but does not affect hemodynamics during induction with double-lumen tubes," Journal of Anesthesia, vol. 24, no. 1, pp. 31-37, 2010.

[34] U. Ozgul, Z. Begec, K. Karahan et al., "Comparison of propofol and ketamine-propofol mixture (ketofol) on laryngeal tube-suction II conditions and hemodynamics: a randomized, prospective, double-blind trial," Current Therapeutic Research, vol. 75, pp. 39-43, 2013.

[35] K. S. Vora, M. S. Prabodhachandran, G. P. Bhosale, N. Singhal, G. P. Parikh, and V. R. Shah, "Comparison of admixtures of propofol-thiopentone, propofol-ketamine and propofol in ambulatory surgery," Journal of Anaesthesiology Clinical Pharmacology, vol. 21, no. 4, pp. 413-418, 2005. 
[36] A. Heffner, D. Swords, J. Kline, and A. Jones, "Frequency and significance of post-intubation hypotension during emergency airway management," Critical Care, vol. 15, no. Suppl 1, p. P154, 2011.

[37] K. Maheshwari, A. Turan, G. Mao et al., "The association of hypotension during non-cardiac surgery, before and after skin incision, with postoperative acute kidney injury: a retrospective cohort analysis," Anaesthesia, vol. 73, no. 10, pp. 1223-1228, 2018.

[38] V. Salmasi, K. Maheshwari, D. Yang et al., "Relationship between intraoperative hypotension, defined by either reduction from baseline or absolute thresholds, and acute kidney and myocardial injury after noncardiac surgery," Anesthesiology, vol. 126, no. 1, pp. 47-65, 2017.

[39] J. B. Bijker, S. Persoon, L. M. Peelen et al., "Intraoperative hypotension and perioperative ischemic stroke after general surgery," Anesthesiology, vol. 116, no. 3, pp. 658-664, 2012.

[40] E. M. Wesselink, T. H. Kappen, H. M. Torn, A. J. C. Slooter, and W. A. van Klei, "Intraoperative hypotension and the risk of postoperative adverse outcomes: a systematic review," British Journal of Anaesthesia, vol. 121, no. 4, pp. 706-721, 2018.

[41] J.-Y. Xu, S.-Q. Ma, C. Pan et al., "A high mean arterial pressure target is associated with improved microcirculation in septic shock patients with previous hypertension: a prospective open label study," Critical Care, vol. 19, no. 1, p. 130, 2015.

[42] K. Maheshwari, B. H. Nathanson, S. H. Munson et al., "The relationship between ICU hypotension and in-hospital mortality and morbidity in septic patients," Intensive Care Medicine, vol. 44, no. 6, pp. 857-867, 2018.

[43] P. Asfar, P. Radermacher, and M. Ostermann, "MAP of 65: target of the past?" Intensive Care Medicine, vol. 44, no. 9, pp. 1551-1552, 2018.

[44] J. W. Yan, S. L. McLeod, and A. Iansavitchene, "Ketaminepropofol versus propofol alone for procedural sedation in the emergency department: a systematic review and meta-analysis," Academic Emergency Medicine, vol. 22, no. 9, pp. 1003-1013, 2015. 